\title{
Fabrication and structural characterization of highly ordered sub-100-nm planar magnetic nanodot arrays over $1 \mathrm{~cm}^{2}$ coverage area
}

\author{
Chang-Peng $\mathrm{Li}^{\mathrm{a})}$ and Igor V. Roshchin \\ Physics Department, University of California, San Diego, La Jolla, California 92093 \\ Xavier Batlle \\ Physics Department, University of California, San Diego, La Jolla, California 92093 \\ and Departament de Física Fonamental, Universitat de Barcelona, 08028 Barcelona, Catalonia, Spain \\ Michel Viret \\ Service de Physique de l'Etat Condensé, DSM/DRECAM-CNRS URA 2464, CEA-Saclay, \\ 91191 Gif-sur-Yvette Cedex, France \\ Frédéric Ott \\ Laboratoire Léon Brillouin CEA/C NRS UMR12, CEA-Saclay, 91191 Gif-sur-Yvette Cedex, France \\ Ivan K. Schuller \\ Physics Department, University of California, San Diego, La Jolla, California 92093
}

(Received 10 March 2006; accepted 11 July 2006; published online 11 October 2006)

\begin{abstract}
Porous alumina masks are fabricated by anodization of aluminum films grown on both semiconducting and insulating substrates. For these self-assembled alumina masks, pore diameters and periodicities within the ranges of 10-130 and 20-200 nm, respectively, can be controlled by varying anodization conditions. $20 \mathrm{~nm}$ periodicities correspond to pore densities in excess of $10^{12}$ per square inch, close to the holy grail of media with $1 \mathrm{Tbit} /$ in. $^{2}$ density. With these alumina masks, ordered sub-100-nm planar ferromagnetic nanodot arrays covering over $1 \mathrm{~cm}^{2}$ were fabricated by electron beam evaporation and subsequent mask lift-off. Moreover, exchange-biased bilayer nanodots were fabricated using argon-ion milling. The average dot diameter and periodicity are tuned between 25 and $130 \mathrm{~nm}$ and between 45 and $200 \mathrm{~nm}$, respectively. Quantitative analyses of scanning electron microscopy (SEM) images of pore and dot arrays show a high degree of hexagonal ordering and narrow size distributions. The dot periodicity obtained from grazing incidence small angle neutron scattering on nanodot arrays covering $\sim 2.5 \mathrm{~cm}^{2}$ is in good agreement with SEM image characterization. (C) 2006 American Institute of Physics.
\end{abstract}

[DOI: 10.1063/1.2356606]

\section{INTRODUCTION}

Nanostructured magnets have attracted great attention recently due to their unique magnetic properties which are completely different from those of continuous films and bulk materials. ${ }^{1-4}$ In addition, nanoscale studies may shed light on the heavily investigated mechanism of exchange bias ${ }^{5-9}$ existing in ferromagnet (FM)-antiferromagnet (AF) bilayers. Fabrication of sub-100-nm FM single layer and FM/AF bilayer nanostructures offers a great opportunity for research on fundamental magnetism at the nanoscale.

A variety of methods are used for nanofabrication, including electron beam and optical lithography. The low throughput and high cost of electron beam lithography lead to limited applicability for the fabrication of nanopatterns with large coverage area. Although optical lithography has high throughput, the smallest size of features that can be produced is limited by the long optical wavelength. Among many nanofabrication methods, ${ }^{10-15}$ a promising technique is based on masks with sub-100-nm self-assembled pores. Such masks, for example, can be fabricated by the anodization of

\footnotetext{
a) Author to whom correspondence should be addressed; electronic mail: changli@physics.ucsd.edu
}

aluminum under appropriate experimental conditions. ${ }^{16-22}$ Arrays of nanopores with tunable pore diameter covering more than $1 \mathrm{~cm}^{2}$ demonstrate the potential application of porous alumina $\left(\mathrm{AlO}_{x}\right)$ masks for nanostructure fabrication. Previously, alumina membranes obtained from the anodization of thick $(>100 \mu \mathrm{m})$ aluminum foils were mostly used for the fabrication of nanowires. ${ }^{23-30}$ These nanowires were grown by electrodeposition, which cannot be easily adapted for the fabrication of nanodots with controlled and uniform thickness. Moreover, this method requires transfer of the alumina membranes onto a substrate. Insufficient adhesion between the membrane and substrate, mask corrugation, delamination, and general transfer problems reduce the reproducibility and uniformity of the fabricated structures. ${ }^{31-35}$

In this work, we fabricate magnetic nanodots using porous masks produced by anodization of $\mathrm{Al}$ films grown directly on substrates. ${ }^{36}$ This mitigates many problems related to transfer of membrane onto the substrate. We are not aware of any previous reports on fabrication of ordered arrays of FM and FM/AF bilayer nanodots over $1 \mathrm{~cm}^{2}$ areas on semiconducting substrates using porous alumina masks. By tuning the anodization parameters, dot arrays with average dot sizes and periodicities of $25-130$ and $45-200 \mathrm{~nm}$, respec- 


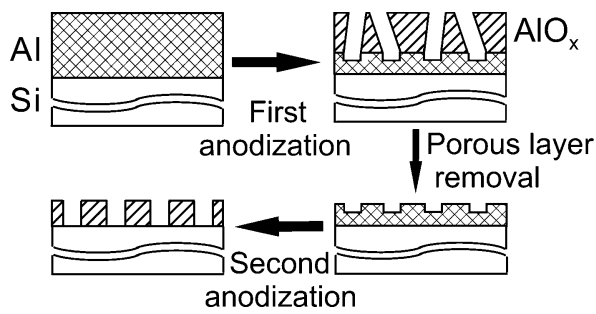

FIG. 1. Schematic of two-step anodization of aluminum film.

tively, are fabricated. The highest nanopore density exceeds $10^{12}$ per square inch, approaching the holy grail of highdensity magnetic media with density of $1 \mathrm{Tbit} / \mathrm{in}^{2}{ }^{2}{ }^{11,37}$

\section{FABRICATION OF SELF-ORDERED POROUS ALUMINA MASKS ON SEMICONDUCTING AND INSULATING SUBSTRATES}

Polished $n$-type (100) Si wafers $(1-10 \Omega \mathrm{cm})$ are used as the semiconducting substrates. The $n$-type silicon substrate is moderately resistant to the acids used in processing and has high enough conductivity to be used as an anode. ${ }^{31}$ The substrate is first ultrasonically cleaned for 15 min using acetone and methanol successively. Then it is etched in $25 \mathrm{wt} \% \mathrm{HF}$ solution for 2 min to remove the native silicon oxide and improve the adhesion between the deposited aluminum film and substrate. Typically, $\sim 6-\mu \mathrm{m}$-thick aluminum layer is deposited by electron beam evaporation (base pressure of $(1-2) \times 10^{-7}$ Torr) from $99.999 \%$ aluminum at a rate of $2 \AA / s$. During deposition, the chamber pressure is $(2-9) \times 10^{-7}$ Torr, and the substrate temperatures are kept between 0 and $25^{\circ} \mathrm{C}$ using liquid nitrogen. The films grown this way have a root-mean-square roughness of $\sim 10 \mathrm{~nm}$, as determined by atomic force microscopy (AFM). Thus, no extra processing is performed prior to anodization.

Improved structural control can be obtained using twostep anodization, ${ }^{32}$ as shown in Fig. 1. The aluminum films are anodized at a constant voltage with $\mathrm{Si}$ substrates as the anodes and a stainless steel plate as the cathode. Voltage and current are monitored during the anodization process. Anodized aluminum layer thickness is calculated from the total charge passing through the anodized area. To obtain the ordered porous patterns with different sizes and periodicities, anodization voltages of $10-80 \mathrm{~V}$ and various electrolytes (sulfuric acid or oxalic acid) are used, as summarized in Table I. Besides the anodization voltage and electrolyte, a variety of other factors affects pore ordering. ${ }^{38-41}$ To enhance the pore self-assembly and ordering the anodization process is slowed down by keeping the electrolyte temperature at

TABLE I. Parameters of aluminum film anodization at $5{ }^{\circ} \mathrm{C}$.

\begin{tabular}{ccc}
\hline \hline $\begin{array}{c}\text { Electrolyte type } \\
(\text { acid })\end{array}$ & $\begin{array}{c}\text { Concentration } \\
(M)\end{array}$ & $\begin{array}{c}\text { Anodization voltage } \\
(\mathrm{V})\end{array}$ \\
\hline Oxalic & 0.05 & $60-80$ \\
Oxalic & 0.3 & $20-60$ \\
Sulfuric & 0.3 & 25 \\
Sulfuric & 1.7 & 19 \\
Sulfuric & 3.9 & 10,15 \\
\hline
\end{tabular}
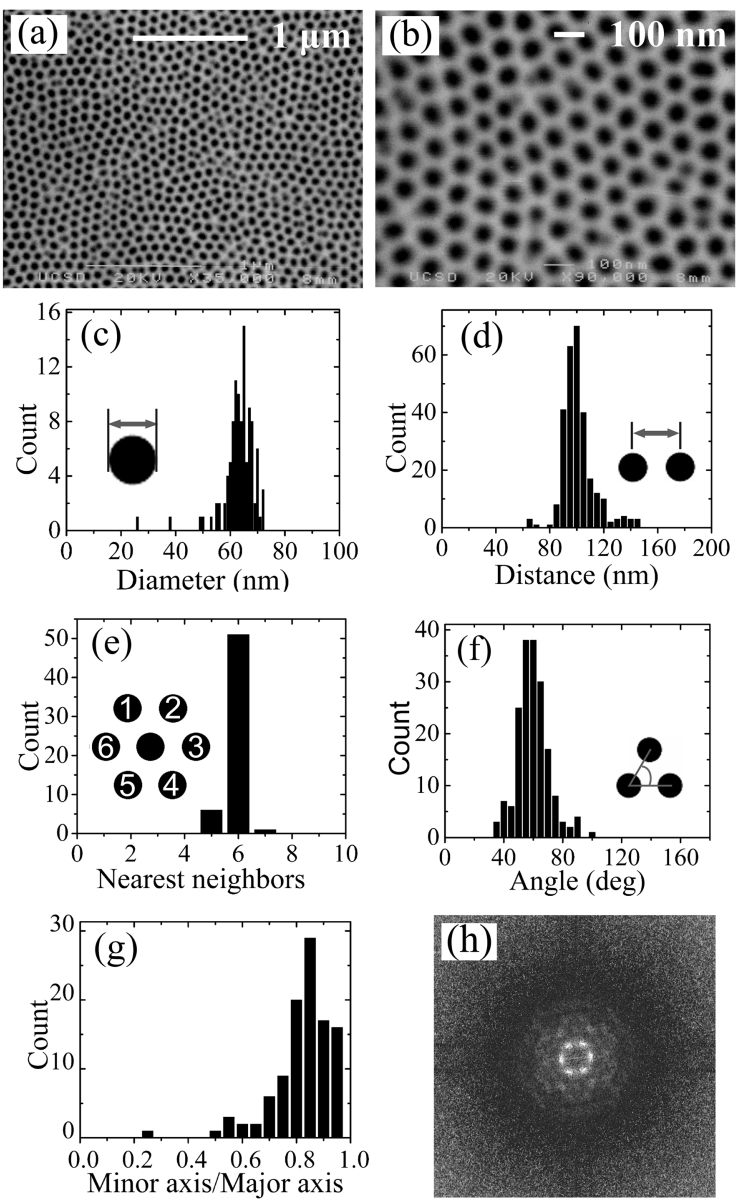

FIG. 2. Typical SEM images of a porous alumina mask (anodized in $0.3 M$ oxalic acid at $40 \mathrm{~V}$ ) with magnifications of (a) 35000 and (b) 90 000; structural characterization of the porous mask shown in (b). (c) Diameter distribution (average of $63 \mathrm{~nm}$ and standard deviation of $6 \mathrm{~nm}$ ). (d) Periodicity distribution (average of $101 \mathrm{~nm}$ and standard deviation of $12 \mathrm{~nm}$ ). (e) Number of nearest-neighbor pores (average of 5.97, standard deviation of 0.18). (f) Angle between the directions to nearest neighbor pores (average of $59.9^{\circ}$, standard deviation of $10.6^{\circ}$ ). (g) Pore shape is analyzed by fitting the pores as ellipses. The average length ratio (minor axis/major axis) of the ellipses is 0.85 , with standard deviation of 0.11 . (h) FFT image of porous structure. The pore periodicity $(\sim 98 \mathrm{~nm})$ calculated from the FFT spot distance agrees well with the result shown in (d). Both the structural characterization and FFT analyses are consistent with a hexagonal ordering of nanopores.

$5^{\circ} \mathrm{C}$. Continuous circulation is used to keep the concentration and temperature of electrolyte uniform. ${ }^{38}$ As a first step, the film is anodized to a certain depth as controlled by the time length. After the first anodization, an aqueous solution with $6.0 \mathrm{wt} \%$ phosphoric acid and $1.8 \mathrm{wt} \%$ chromic acid at $60^{\circ} \mathrm{C}$ is used to selectively remove the porous alumina layer, without affecting the aluminum layer below. As a result, a regular dimple pattern is obtained on the intact, nonoxidized aluminum layer. This aluminum layer determines the final mask thickness. This must be thick enough to ensure a good mask lift-off after the deposition of magnetic materials. However, if the mask thickness (pore height) is much larger than the pore diameter, the bottom of the pores becomes inaccessible to the evaporated materials ("shadow effect"). Details of the shadow effect will be discussed in the subsequent magnetic nanodot fabrication section. In the second step, the patterned aluminum is anodized completely using 
TABLE II. Summary of structural characterization of typical nanopore arrays in anodized alumina.

\begin{tabular}{|c|c|c|c|c|c|c|}
\hline $\begin{array}{c}\text { Electrolyte } \\
\text { (acid) }\end{array}$ & $\begin{array}{l}\text { Anodization } \\
\text { voltage }(\mathrm{V})\end{array}$ & $\begin{array}{c}\text { Pore } \\
\text { widening } \\
\text { time }(\min )\end{array}$ & $\begin{array}{l}\text { Diameter } \\
(\mathrm{nm})\end{array}$ & $\begin{array}{l}\text { Periodicity } \\
(\mathrm{nm})\end{array}$ & $\begin{array}{c}\text { Number of } \\
\text { nearest } \\
\text { neighbors }\end{array}$ & $\begin{array}{l}\text { Angle between } \\
\text { directions to } \\
\text { nearest neighbors } \\
\text { (deg) }\end{array}$ \\
\hline $0.3 M$ & 40 & 80 & $63 \pm 6$ & $101 \pm 12$ & $6.0 \pm 0.2$ & $59.9 \pm 10.6$ \\
\hline \multicolumn{7}{|l|}{ Oxalic } \\
\hline $0.3 M$ & 25 & 45 & $29 \pm 6$ & $59 \pm 7$ & $5.9 \pm 0.6$ & $58.8 \pm 10.4$ \\
\hline \multicolumn{7}{|l|}{ Sulfuric } \\
\hline $1.7 M$ & 19 & 30 & $24 \pm 3$ & $43 \pm 6$ & $5.9 \pm 0.6$ & $58.1 \pm 12.8$ \\
\hline Sulfuric & & & & & & \\
\hline
\end{tabular}

the same conditions as the first anodization process, with pores nucleating in the dimples. The ordering and control of the arrays obtained by this method are competitive with the much more expensive and technically demanding stamping process. $^{31}$

After anodization of the aluminum film, an alumina "barrier" layer, with thickness proportional to the anodization voltage, is formed at the bottom of pores. The thickness of the barrier layer can be decreased by gradually reducing the anodization voltage to one-fourth of the original voltage after the anodization reaches the $\mathrm{Si}$ substrate, which is indicated by a sharp decrease of anodization current. ${ }^{25}$ Subsequent $5 \mathrm{wt} \%$ phosphoric acid etching is used to remove the alumina barrier layer. At the same time, the pores are widened at $8 \mathrm{~nm} / \mathrm{h}$, with their periodicity unaffected. After the removal of barrier layer, extra phosphoric acid etching can be used to widen pores further. The pore opening at the top can also be reduced by depositing aluminum or silver on top of the alumina mask at an angle of $60^{\circ}$ with the normal as confirmed by the reduced diameter of Fe dots fabricated using this mask.

The porous alumina masks are imaged using scanning electron microscopy (SEM) after each stage of fabrication. An accelerating voltage of $20 \mathrm{kV}$ and a probe current of $3.0 \times 10^{-9} \mathrm{~A}$ are used to minimize charging of the sample while providing high enough resolution. Two SEM images of a typical porous alumina mask anodized at $40 \mathrm{~V}$ in $0.3 \mathrm{M}$ oxalic acid are shown in Figs. 2(a) and 2(b). To characterize the porous structures, grayscale SEM images are converted to binary black-and-white images. The pixels with grayscale values exceeding a threshold are set to black; all others are set to white. The threshold values are manually chosen so that the black areas in the black-and-white images reproduce the pores in the original grayscale images. NIH IMAGEJ software package is used to measure the sizes, shapes, and center positions of pores from the black-white images. ${ }^{42}$ Pore periodicity (center to center distance between nearest-neighbor pores), number of nearest-neighbor pores, and angle between directions to neighboring pores are calculated from the positions of pore centers. Analysis of the image in Fig. 2(b) shows that the pores have an average diameter of $63 \mathrm{~nm}$ and an average periodicity of $101 \mathrm{~nm}$, with a standard deviation for both less than 10\% [Figs. 2(c) and 2(d)]. The number of nearest-neighbor pores (average of 5.97) and angle between the directions to neighboring pores (average of 59.9 $)$ are consistent with a regular hexagonal arrangement [Figs. 2(e) and 2(f)]. Here, we do not include the pores close to the image boundary since they do not have all nearest neighbors within the image. The pore shapes are analyzed by fitting them as ellipses. The average length ratio (minor axis/major axis) of the ellipses is 0.85 [Fig. 2(g) ]. Figure 2(h) shows the two-dimensional (2D) fast Fourier transform (FFT) image of Fig. 2(a). The six spots confirm the regular hexagonal arrangement of pores. The pore periodicity $(\sim 98 \mathrm{~nm})$ calculated from the FFT spot distance agrees well with the result shown in Fig. 2(d). The porous mask in this work exhibits a lower degree of regularity than earlier self-supporting alumina membranes due to the smaller thickness of the aluminum film used in this paper compared with the severalhundred-micron-thick aluminum foils used in Refs. 16-19. For thicker aluminum layers, a higher order and narrower pore size distribution is found with the same anodization conditions. Further improvements in ordering, required for industrial applications, can be achieved by additional prestamping of the aluminum surface. ${ }^{31}$

During the anodization process, pore periodicity and diameter are proportional to the anodizing voltage. ${ }^{39}$ To obtain masks with small pore size, low anodization voltage and concentrated sulfuric acid (up to 3.9M) are used. Despite its low reactivity to acid etching, $n$-type $\mathrm{Si}$ is etched slightly by the concentrated sulfuric acid solutions, which causes the anodized alumina mask to peel off. The etching of the substrate has been confirmed by anodizing two identical aluminum films in oxalic acid and sulfuric acid. After the anodization reaches the substrate, the mask anodized in sulfuric acid peels off with further anodization ("overanodization"). With the same anodizing voltage and temperature, the mask remains intact when overanodized in oxalic acid. Because of this, the anodization with sulfuric acid must be stopped as soon as it reaches the silicon substrate. With the anodization parameters in Table I and subsequent pore size adjustments, porous masks with pore diameters of $10-135 \mathrm{~nm}$ and periodicities of 20-200 $\mathrm{nm}$ are fabricated. The results of structural analyses of typical alumina masks anodized in $0.3 M$ oxalic, $0.3 \mathrm{M}$ sulfuric, and $1.7 \mathrm{M}$ sulfuric, at constant anodization voltages of 40, 25, and $19 \mathrm{~V}$, respectively, are shown in Table II. A typical structural domain size of the hexagonally ordered pores is $\sim 0.5-1 \mu \mathrm{m}$. Masks with pore periodicity of $20 \mathrm{~nm}$ (Fig. 3) exhibit pore density in excess of $10^{12}$ per square inch, which suggests possible applications of this fabrication method for ultrahigh-density recording media. 


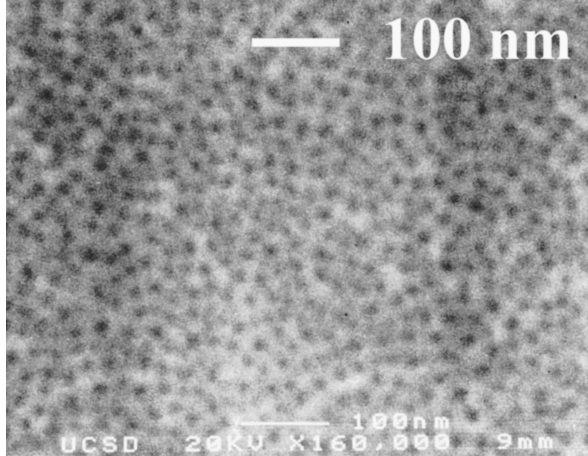

FIG. 3. Typical SEM image of an alumina mask anodized at $10 \mathrm{~V}$ in $3.9 M$ sulfuric acid with pore density exceeding $10^{12}$ per square inch.

To fabricate alumina masks on insulating substrates, 200-nm-thick aluminum films are deposited on sapphire substrates coated with 20 -nm-thick gold films. The gold film is used as the anode in the aluminum anodization process. ${ }^{36,43}$ To avoid mask delamination, the anodization process must be stopped as soon as it reaches the gold film, which is indicated by a sharp increase of anodization current. This current increase becomes less abrupt for thicker aluminum films, probably due to nonuniformity of the anodization rate over the film surfaces. Thus, it is more difficult to determine the time at which the anodization process reaches the gold film for thicker aluminum films. Such a limitation in the aluminum film thickness results in a lower degree of regularity compared to that of the masks on Si substrates.

\section{FABRICATION OF MAGNETIC DOT ARRAYS USING POROUS ALUMINA MASKS}

In this section, we discuss the fabrication of single FM layer and FM/AF bilayer nanodot arrays using porous alumina masks. Before magnetic materials deposition, the porous alumina mask is baked in situ at $500^{\circ} \mathrm{C}$ for $1 \mathrm{~h}$ to remove water and hydrocarbons inside the pores. To fabricate single FM layer dots, $20 \mathrm{~nm}$ of the ferromagnet $(\mathrm{Fe}, \mathrm{Ni}$, and $\left.\mathrm{Fe}_{20} \mathrm{Ni}_{80}\right)$ are deposited at a rate of $1 \AA / \mathrm{s}$ on a sample with a porous mask, kept at $200^{\circ} \mathrm{C}$, using electron beam evaporation. With the sample at $150^{\circ} \mathrm{C}$, a 5-8 nm thick silver capping layer is deposited to protect the dots from oxidation. After mask lift-off in either 10 wt $\% \mathrm{NaOH}$ solution at room temperature for $2 \mathrm{~min}$ or in a mixture of $6.0 \mathrm{wt} \%$ phosphoric acid and $1.8 \mathrm{wt} \%$ chromic acid at $60^{\circ} \mathrm{C}$ for 15 min, we obtain single layer FM dot arrays (Fig. 4). To check the adhesion of the FM dots to the Si substrates, we sonicated a typical Fe dot sample in acetone for $10 \mathrm{~min}$. SEM images of this sample indicate that the dots are intact, which confirms the high stability of FM dots fabricated by this method on the Si substrate.

Arrays of FM/AF bilayer dots for nanoscale exchange bias studies are fabricated using about 300-nm-thick porous alumina masks, as described above. After deposition of $20 \mathrm{~nm}$ of $\mathrm{FM}\left(\mathrm{Fe}, \mathrm{Ni}\right.$, and $\mathrm{Fe}_{20} \mathrm{Ni}_{80}$ ), the sample temperature is increased to $250{ }^{\circ} \mathrm{C}$ and $50 \mathrm{~nm} \mathrm{AF}\left(\mathrm{FeF}_{2}\right)$ is deposited at a rate of $1 \AA / \mathrm{s}$. However, the aforementioned mask lift-off procedure cannot be used for the $\mathrm{FM} / \mathrm{FeF}_{2}$ bilayer dot arrays because $\mathrm{FeF}_{2}$ is soluble in the aqueous etchant solution. In-

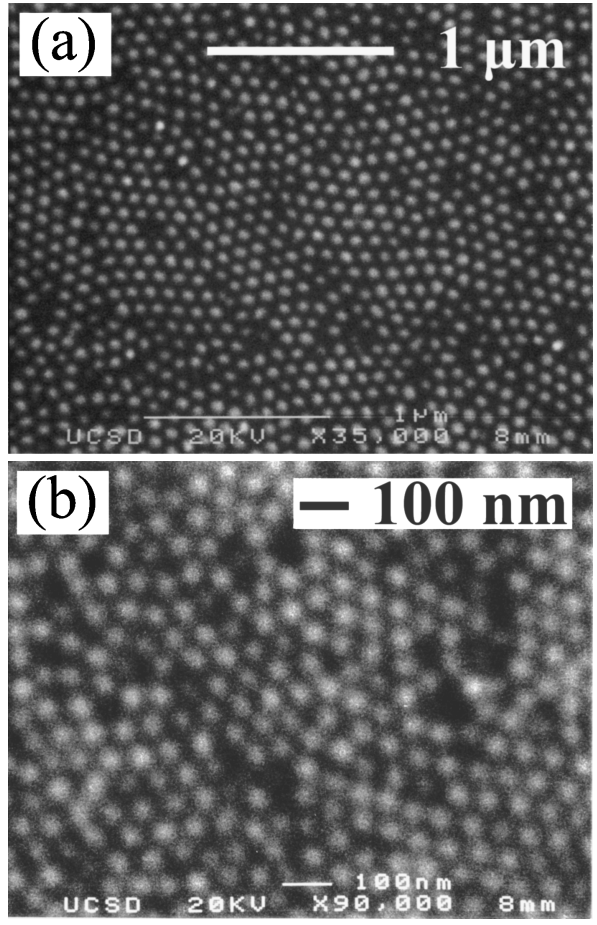

FIG. 4. (a) Typical SEM image of Fe dot array (fabricated using alumina mask anodized at $40 \mathrm{~V}$ ) with average diameter and periodicity of 67 and $104 \mathrm{~nm}$, respectively; (b) typical SEM image of Fe dot array (fabricated using alumina mask anodized at $25 \mathrm{~V}$ ) with average diameter and periodicity of 32 and $63 \mathrm{~nm}$, respectively.

stead, all the magnetic layers on top of the mask are removed using $500 \mathrm{eV}$ argon-ion milling at an angle of $45^{\circ}$, as sketched in Fig. 5. The sample is rotated in plane to ensure uniform milling. After $\sim 8 \mathrm{~min}$ ion milling, all the materials on top of the mask and about top $50 \mathrm{~nm}$ of alumina mask are removed, but the materials at the bottom of the pores, protected by the alumina mask, are left intact. This is accomplished by ensuring that the distance between the top of the alumina mask and the surface of the magnetic material at the bottom of pores is larger than the pore diameter during the entire ion milling process. In this fashion the magnetic bilayer dot array is embedded in the nonmagnetic porous alumina masks.

To verify the successful fabrication of $\mathrm{FM} / \mathrm{FeF}_{2}$ bilayer dots, the sample is measured using superconducting quantum

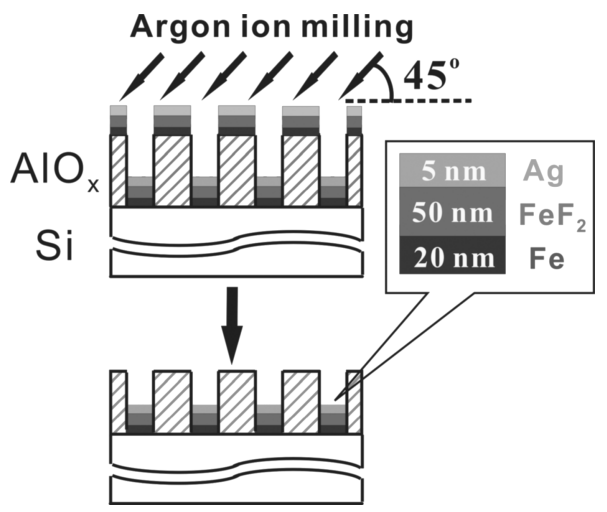

FIG. 5. Schematic of fabrication process of $\mathrm{FM} / \mathrm{FeF}_{2}$ exchange-biased bilayer nanodot array using argon-ion milling at an angle of $45^{\circ}$. 
interference device (SQUID) magnetometry before and after the argon-ion milling. Observation of exchange bias (over $100 \mathrm{Oe}$ at $10 \mathrm{~K}$ ) up to $\sim 80 \mathrm{~K}$ (Néel temperature for $\mathrm{FeF}_{2}$ ) confirms the presence of a clean interface between the FM and $\mathrm{FeF}_{2}$ layers. The measured magnetic moment in saturation is proportional to the FM volume. The ratio of the FM volume on top of the mask to its total volume should be equal to the ratio of the area outside the pores to the total mask area (typically around 70\%). Reduction of the magnetic moment after the ion milling in the same proportion confirms the successful removal of the magnetic layers from the top of the mask. As the sample is ion milled further, no additional reduction of magnetic moment and exchange bias are found. This indicates that the ion milling does not affect neither the FM nor the $\mathrm{FeF}_{2}$ at the bottom of pores.

While the top of the FM is protected, its sides are exposed and may be oxidized. For continuous FM films or dots that are larger than $100 \mathrm{~nm}$, formation of magnetic metal oxide has only a small effect on the magnetic properties, such as exchange bias. However, the magnitude of exchange bias of nanodots is proportional to the ratio of the oxidized FM surface to the FM volume; i.e., the magnitude of exchange bias increases as the dot size decreases. Thus, the effect of the oxidation must be seriously considered for sub100-nm magnetic nanodots. To avoid dot oxidation, a thick aluminum layer is deposited ex situ immediately after ion milling or mask lift-off. With this protective layer, there is no measurable effect attributable to FM oxidation, indicating that the amount of metal oxide formed in this case is negligible.

When the magnetic dots are fabricated using mask liftoff, the phosphoric acid etch before magnetic materials deposition should be long enough to remove the alumina barrier layer remaining at the bottom of pores. Otherwise, the dots would also be removed during subsequent mask lift-off. On the other hand, with the argon-ion milling, the barrier layer does not constitute a problem. Thus, shorter etching times resulting in smaller pore diameters can be used. Hence, the range of diameters of dots that can be fabricated with the same periodicity is wider.

As mentioned earlier, porous alumina masks used for fabrication of dot arrays should be thin enough to avoid the shadow effect. To study the dependence of the shadow effect on mask thickness, $\mathrm{Fe}$ is deposited through porous alumina masks with 65-nm-diameter pores and 1- and $0.4-\mu \mathrm{m}$-thicknesses. After mask lift-off, few dots are found on the sample with the $1-\mu$ m-thick alumina mask, while a typical ordered dot array is found with the $0.4-\mu \mathrm{m}$-thick alumina mask. Thus, to fabricate dots using a porous mask, an appropriate pore aspect ratio (diameter per thickness) must be chosen. For example, a $0.12 \mu \mathrm{m}$ or thinner alumina mask must be chosen to fabricate 20-nm-diameter dots, while $0.4 \mu \mathrm{m}$ alumina mask can be used to obtain 65 -nm-diameter dots. As discussed earlier, the porous mask thickness is determined by the alumina layer thickness remaining intact after the first anodization. Nonuniformity of the anodization rate over the aluminum surface complicates fabrication of thin masks from very thick aluminum films. Hence, the high-

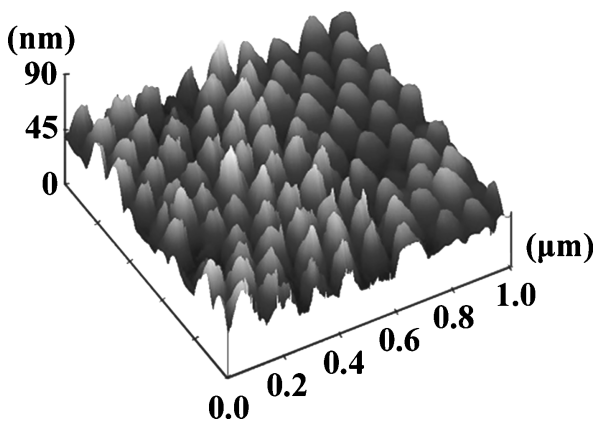

FIG. 6. AFM image of a typical Fe dot array (fabricated using alumina mask anodized at $40 \mathrm{~V}$ ). The standard deviation of the dot height is about $4 \mathrm{~nm}$.

est density of the dot arrays reproducibly produced so far is about $0.4 \times 10^{12}$ per square inch, despite the highest nanopore density being $1 \times 10^{12}$ per square inch.

The nanodot arrays are characterized from analyses of SEM images (Fig. 4), as is done for nanopore arrays. These analyses show that the fabricated nanodots are circular and have a hexagonal arrangement with narrow size and periodicity distributions. A comparison of the size and periodicity distributions for a porous mask to those distributions for the dot array fabricated with this mask indicates a good pattern transfer. Nanodot structures are also investigated by AFM, which yields a close agreement with the SEM analyses. Moreover, AFM measurement shows that the standard deviation of dots height is about $4 \mathrm{~nm}$ (Fig. 6). Alumina masks with various sizes and periodicities allow the average dot diameter and periodicity to be tuned from 25 to $130 \mathrm{~nm}$ and from 45 to $200 \mathrm{~nm}$, respectively. For these arrays (20-nm-thick dots with dot periodicity about twice the dot diameter) the interaction between the dots can be neglected. ${ }^{44-46}$ Since the $10 \%$ standard deviation in the dot size and periodicity and the $4 \mathrm{~nm}$ standard deviation in the dot height are relatively small, magnetic measurements of such a dot array can be used to investigate the magnetism of a single noninteracting dot.

Since these nanodot arrays cover a macroscopically large area (over $1 \mathrm{~cm}^{2}$ ), their magnetic properties can be investigated using techniques that require relatively large quantities of material including SQUID and vibrating sample magnetometry, polarized neutron scattering, ferromagnetic resonance, etc. For example, grazing incidence small angle neutron scattering (GISANS), performed at the Laboratoire Leon Brillouin (LLB, Saclay) spectrometer PAPOL with $8 \AA$ wavelength, is used to analyze the microstructure of the samples. We are not aware of any neutron scattering experiments performed on sub-100-nm magnetic dots previously. The geometry is such that the scattering plane $(X, Z)$ is formed by the transmitted and reflected beams [Fig. 7(a)]. Figure 7(b) shows the scattering intensity as the function of momentum transfer vector $Q_{y}$ for a continuous 20-nm-thick film and a $20-\mathrm{nm}$-thick Fe dot sample with $65 \mathrm{~nm}$ average diameter. The statistical errors are given by the square root of the scattering intensity. Due to their small sizes, most of the error bars are covered by symbols. The peak at $Q_{y}=0$ corresponds to the specular reflection and appears in the neutron scattering of both dots and film samples. The extra shoulders 

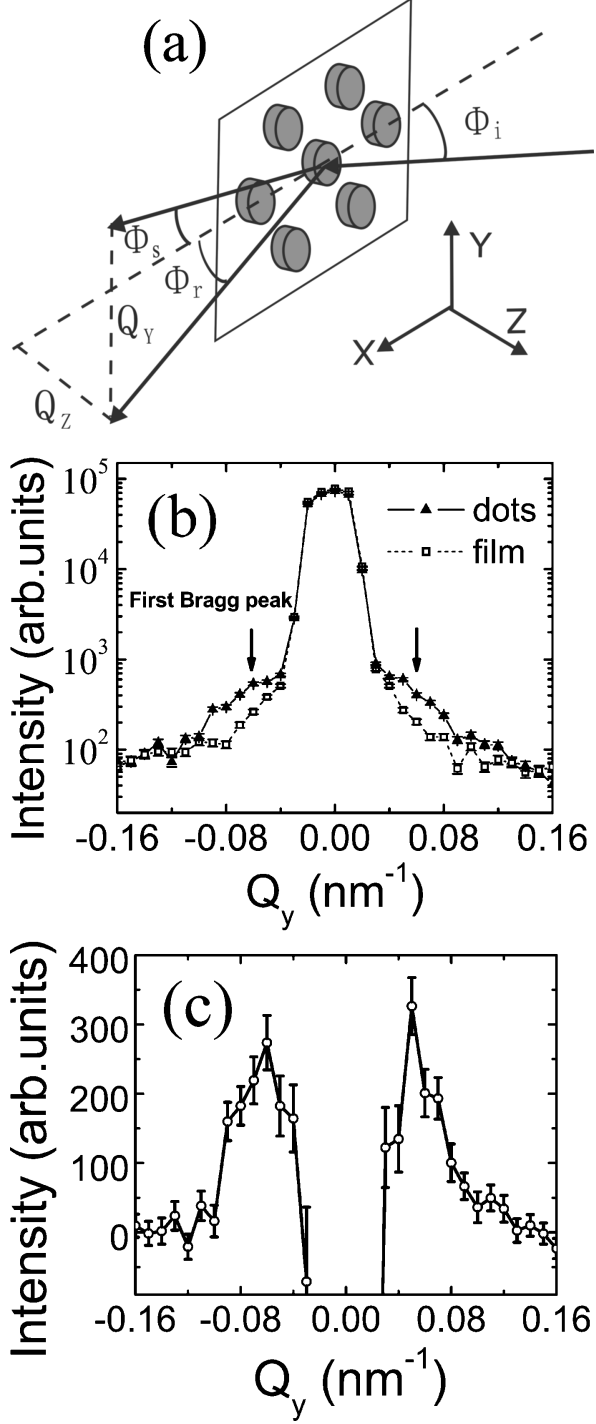

FIG. 7. (a) Geometry of GISANS measurement. (b) Scattering intensity as the function of momentum transfer vector $Q_{y}$ for an array of Fe dots $(20 \mathrm{~nm}$ height, $65 \mathrm{~nm}$ average diameter) $(\boldsymbol{\Delta})$ and a continuous Fe film of the same thickness $(\square)$. The statistical errors are given by the square root of the scattering intensity. Due to their small sizes, most of the error bars are covered by symbols. (c) Difference between the scattering intensity of dots and that of the film.

appearing for the dots are the first Bragg peaks due to the spatial ordering of the dots. These peaks are even more evident in Fig. 7(c), which shows the difference between the scattering intensity of the dots and that of the film. The dip [not fully shown in Fig. 7(c)] at the specular angle, $Q_{y}=0$, is due to the transfer of the scattering intensity from the specular reflection peak into the Bragg peaks in the case of the dots. Positions of these Bragg peaks correspond to a dot periodicity of $\sim 100 \mathrm{~nm}$. It is noteworthy that this value of periodicity is observed from the entire $\left(\sim 2.5 \mathrm{~cm}^{2}\right.$ in area) sample. This periodicity is in good agreement with that obtained from the SEM image $(104 \mathrm{~nm})$, indicating that the variation of the periodicity over the sample area is small. This also validates our characterization based on the SEM images of small typical areas of the sample.

\section{CONCLUSIONS}

Porous alumina masks are fabricated by anodization of aluminum films grown on semiconducting and insulating substrates. Control of the anodizing voltage and electrolyte allows fabrication of self-assembled porous alumina masks with diameters of $10-130 \mathrm{~nm}$ and periodicities of $20-250 \mathrm{~nm}$. Masks with periodicity of $20 \mathrm{~nm}$ exhibit pore density exceeding $10^{12}$ per square inch, which might have relevance for magnetic media with the density of $1 \mathrm{Tbit} / \mathrm{in}^{2}$. With these alumina masks, ordered FM nanodot arrays with coverage exceeding $1 \mathrm{~cm}^{2}$ are fabricated by electron beam evaporation and subsequent mask lift-off. For $\mathrm{FM} / \mathrm{FeF}_{2}$ bilayer samples, argon-ion milling at an angle of $45^{\circ}$ is used instead of the mask lift-off. The average dot size and periodicity can be tuned in the $25-130$ and $45-250 \mathrm{~nm}$ ranges, respectively. Dot periodicity obtained from small angle neutron scattering on nanodot arrays covering $\sim 2.5 \mathrm{~cm}^{2}$ is in good agreement with the SEM characterizations.

\section{ACKNOWLEDGMENTS}

Support by the Air Force Office of Scientific Research is gratefully acknowledged. One of the authors (X.B.) acknowledges funding from the Spanish MECD (PR20030149) and CICYT (MAT2003-01124), and the Catalan DURSI (ACI2002-005). The authors thank Bernd Fruhberger, Amos Sharoni, and Corneliu Colesniuc for their help with the argon-ion milling, George Kassabian for his help in building the electronic system for automated control of anodization, and Casey Miller and Casey Chiang for a critical reading of this manuscript.

${ }^{1}$ F. J. Himpsel, J. E. Ortega, G. J. Mankey, and R. F. Willis, Adv. Phys. 47, 511 (1998).

${ }^{2}$ R. Skomski, J. Phys.: Condens. Matter 15, R841 (2003).

${ }^{3}$ X. Batlle and A. Labarta, J. Phys. D 35, R15 (2002).

${ }^{4}$ C. E. Zaspel, B. A. Ivanov, J. P. Park, and P. A. Crowell, Phys. Rev. B 72, 024427 (2005).

${ }^{5}$ A. P. Malozemoff, J. Appl. Phys. 63, 3874 (1988).

${ }^{6}$ U. Nowak and K. D. Usadel, Phys. Rev. B 66, 014430 (2002).

${ }^{7}$ J. Nogués and I. K. Schuller, J. Magn. Magn. Mater. 192, 203 (1999).

${ }^{8}$ A. E. Berkowitz and K. Takano, J. Magn. Magn. Mater. 200, 552 (1999).

${ }^{9}$ J. Eisenmenger, Z.-P. Li, W. A. A. Macedo, and I. K. Schuller, Phys. Rev. Lett. 94, 057203 (2005).

${ }^{10}$ J. I. Martín, J. Nogués, K. Liu, J. L. Vicent, and I. K. Schuller, J. Magn. Magn. Mater. 256, 449 (2003).

${ }^{11}$ B. D. Terris and T. Thomson, J. Phys. D 38, R199 (2005).

${ }^{12}$ M. Geissler and Y. Xia, Adv. Mater. (Weinheim, Ger.) 16, 1249 (2004).

${ }^{13}$ C. A. Ross et al., J. Vac. Sci. Technol. B 17, 3168 (1999); C. A. Ross, Annu. Rev. Mater. Res. 31, 203 (2001).

${ }^{14}$ L. J. Heyderman, H. H. Solak, C. David, D. Atkinson, R. P. Cowburn, and F. Nolting, Appl. Phys. Lett. 85, 4989 (2004).

${ }^{15}$ R. O. Valles, M. S. Lund, C. Leighton, and M. A. Hillmyer, J. Mater. Chem. 14, 2729 (2004).

${ }^{16} \mathrm{H}$. Masuda and K. Fukuda, Science 268, 1426 (1995).

${ }^{17}$ H. Chik and J. M. Xu, Mater. Sci. Eng., R. 43, 103 (2004).

${ }^{18}$ H. Masuda, K. Yada, Y. Sakamoto, and A. Osaka, Jpn. J. Appl. Phys., Part 2 37, L1340 (1998).

${ }^{19}$ H. Masuda, F. Hasegwa, and S. Ono, J. Electrochem. Soc. 144, L127 (1997).

${ }^{20}$ J. P. O'Sullivan and G. C. Wood, Proc. R. Soc. London, Ser. A 317, 511 (1970).

${ }^{21}$ I. Serebrennikova, P. Vanysek, and V. I. Birss, Electrochim. Acta 42, 145 (1997).

${ }^{22}$ S. K. Thamida and H. C. Chang, Chaos 12, 240 (2002).

${ }^{23}$ C. Z. Wang, G. W. Meng, Q. Q. Fang, X. S. Peng, Y. W. Wang, Q. Fang, 
and L. D. Zhang, J. Phys. D 35, 738 (2002).

${ }^{24}$ G. Schmid, J. Mater. Chem. 12, 1231 (2002).

${ }^{25}$ G. Sauer, G. Brehm, S. Schneider, K. Nielsch, R. B. Wehrspohn, J. Choi, H. Hofmeister, and U. Gösele, J. Appl. Phys. 91, 3243 (2002).

${ }^{26}$ H. Zeng, R. Skomski, L. Menon, Y. Liu, S. Bandyopadhyay, and D. J. Sellmyer, Phys. Rev. B 65, 134426 (2002).

${ }^{27}$ K. H. Lee, H. Y. Lee, W. Y. Jeung, and W. Y. Lee, J. Appl. Phys. 91, 8513 (2002).

${ }^{28}$ K. Nielsch, R. B. Wehrspohn, J. Barthel, J. Kirschner, and U. Gösele, Appl. Phys. Lett. 79, 1360 (2001).

${ }^{29}$ Y. Peng, H. L. Zhang, S. L. Pan, and H. L. Li, J. Appl. Phys. 87, 7405 (2000).

${ }^{30}$ M. Zheng, L. Menon, H. Zeng, Y. Liu, S. Bandyopadhyay, R. D. Kirby, and D. J. Sellmyer, Phys. Rev. B 62, 12282 (2000).

${ }^{31}$ H. Masuda, K. Yashi, Y. Sakamoto, M. Nakao, T. Tamamura, and K. Nishio, Jpn. J. Appl. Phys., Part 2 40, L1267 (2001).

${ }^{32}$ H. Masuda and M. Satoh, Jpn. J. Appl. Phys., Part 2 35, L126 (1996).

${ }^{33}$ S. Shingubara, O. Okino, Y. Murakami, H. Sakaue, and T. Takahagi, J. Vac. Sci. Technol. B 19, 1901 (2001).

${ }^{34}$ D. Crouse, Y. H. Lo, A. E. Miller, and M. Crouse, Appl. Phys. Lett. 76, 49 (2000).

${ }^{35}$ K. Liu, J. Nogués, C. Leighton, I. V. Roshchin, H. Masuda, K. Nishio, and
I. K. Schuller, Appl. Phys. Lett. 81, 4434 (2002); J. H. Choi, T.-H. Kim, J. Seo, and Y. Kuk, ibid. 85, 3235 (2004).

${ }^{36}$ M. S. Sander and L. S. Tan, Adv. Funct. Mater. 13, 393 (2003).

${ }^{37}$ R. W. Wood, J. Miles, and T. Olson, IEEE Trans. Magn. 38, 1711 (2002), M. H. Kryder and R. W. Gustafson, J. Magn. Magn. Mater. 287, 449 (2005).

${ }^{38}$ O. Jessenky, F. Müller, and U. Gösele, J. Electrochem. Soc. 145, 3735 (1998).

${ }^{39}$ A. P. Li, F. Müller, A. Birner, K. Nielsch, and U. Gösele, J. Appl. Phys. 84, 6023 (1998).

${ }^{40}$ L. Ba and W. S. Li, J. Phys. D 33, 2527 (2000).

${ }^{41}$ R. L. Chiu, P. H. Chang, and D. H. Tung, Thin Solid Films 260, 47 (1995).

${ }^{42}$ M. D. Abramoff, P. J. Magelhaes, and S. J. Ram, Biophotonics Int. 11, 36 (2004).

${ }^{43}$ Y. Yang, H. L. Chen, Y. F. Mei, J. B. Chen, X. L. Wu, and X. M. Bao, Solid State Commun. 123, 279 (2002).

${ }^{44}$ J. Mejía-López, D. Altbir, A. H. Romero, X. Batlle, I. V. Roshchin, C.-P. Li, and I. K. Schuller, J. Appl. Phys. (accepted).

${ }^{45}$ K. Y. Guslienko, Appl. Phys. Lett. 75, 394 (1999).

${ }^{46}$ A. J. Bennett and J. M. Xu, Appl. Phys. Lett. 82, 2503 (2003). 